\title{
Pathologic changes in pig organs, infected with the Aujeszky's disease
}

\author{
V.V. Ukhovskyi ${ }^{1}$, O.M. Romanov ${ }^{2}$, B.V Borisevich ${ }^{3}$, O.A. Tarasov ${ }^{2}$, T.M. Ukhovska ${ }^{1}$, \\ A.V. Pyskun', V.V. Solomon ${ }^{3}$, O.Y. Ayshpur ${ }^{2}$ \\ ${ }^{1}$ State Scientific Research Institute of Laboratory Diagnostic and Veterinary Sanitary Expertise, \\ 30 Donetska St, Kyiv, 03151, Ukraine \\ ${ }^{2}$ Institute of Veterinary Medicine, NAAS of Ukraine, \\ 30 Donetska St, Kyiv, Ukraine, 03151 \\ ${ }^{3}$ National University of Life and Environmental Sciences of Ukraine, Kyiv, Ukraine, \\ 15 Heroyiv Oborony St, Kyiv, Ukraine, 03041
}

Corresponding author E-mail: uhovskiy@ukr.net

Received: 03.09.2020. Accepted: 03.10.2020

\begin{abstract}
The data on histological studies of organs of suckling piglets that died from Aujeszky's disease presented in the article. To study of the microscopic changes, organ and tissue samples were taken from 5 suckling pigs (aged 4 to 6 days old). In all piglets, the diagnosis was confirmed by polymerase chain reaction. For histological studies, tissue samples were taken from the following organs: lungs, stomach, intestines, liver, pancreas, kidneys, thymus, lymph nodes, spleen, brain and spinal cord. The studies established that as a result of the Aujeszky's disease virus impact on various organs and tissues, in dead pigs, significant pathomorphological changes are observed in most parenchymal organs. As a result of the histological studies of the internal organs of the pigs, died due to Aujeszky's disease the presence of significant microscopic changes in all morphological formations of these organs were found. It was described a number of micromorphological signs of piglets pathological changes and a set of criteria that can be used to substantiate a postmortem diagnosis of this disease was identified.
\end{abstract}

Keywords: Aujeszky's disease; Suis Herpes Virus 1; Piglets; Diagnostics; Polymerase chain reaction; Microscopic changes.

\section{Introduction}

Aujeszky's disease (pseudo-rabies) is contagious disease with acute course occurring in the form of epizootics and sporadic cases. It causes significant economic losses to livestock holders, especially in countries with intensively developed pig and fur farming (Gu et al., 2018; Ukhovskyi et al., 2020; Xia et al., 2018). The disease is caused by the Aujeszky's disease virus (Suid Herpesvirus 1), which belongs to the Herpesviridae family, the Alphaherpesvirinae subfamily, the Varicellovirus genus (Dong et al., 2017).

Economic impact of Aujeszky's disease consists of the death of animals, sanitary slaughtering, a decrease in animal growth, loss of fertility, culling of mascara, abortion, elimination costs, costs for general and specific prevention of the disease (Liu et al., 2019).

Lethal cases of the disease are observed mainly in piglets, but in adult pigs the disease usually accompanied by the long-term or lifelong virus persistence. The mortality rate of newborn piglets reaches $90-100 \%$, and in one month old - up to $60 \%$ (Verpoest et al., 2016). With the increasing age of animals, the number of manifestations of clinical signs decreases. In finishing and breeding pigs, the disease is most often characterized by respiratory syndrome (cough, shortness of breath, fever, upper respiratory tract and lungs) (Xia et al., 2018). In sows, the disease is characterized by altered reproduction (stillbirth, mummification and fetal mortality, infertility) (Verpoest et al., 2016). The reconvalescent pigs of Aujeszky's disease became the main reservoir of the virus and acting as the source of infection (Tong et al., 2015; Zhao et al., 2018). Due to the variety of forms of the course of the disease, laboratory confirmation of the diagnosis is a prerequisite for suspected Aujeszky's disease in all cases (Dong et al., 2017).

As a result of the virus impact on various organs in animals that have died or been ill with Aujeszky's disease, significant pathological changes were observed in magority of a parenchymal organs (Lai et al., 2019; Tong et al., 2020). According to the reports by a number of authors (Cano-Terriza et al., 2019; Deblanc et al., 2019), after spreading through the mucous membranes of the oral cavity or upper respiratory tract, the virus accumulates mainly in the brain, its reproduction in this organ leads to the development of non-suppurative lymphacytic encephalitis with a nervous syndrome as a result of pathogenesis. When the virus penetrates the skin, its enhanced reproduction occurs at the infected area with changes of the blood chemical composition with increasing of the acetylcholine and histamine contents in the central nervous system and skin changes, causing the development of hyperesthesia (itching) (Miller et al., 2019; Sorooshyari et al., 2018 ; Wong et al., 2019). In pig organism, on the contrary, changes are more often localized in the lungs (Lane et al., 2012; Laval et al., 2018) and abdominal cavity (Tong et al., 2020; Verpoest et al., 2016), less often in the central nervous system. (Guan et al., 2018; Sorooshyari et al., 2018), this is due to the fact that the virus rarely penetrate the blood-brain barrier (Miller et al., 2019). Because of that clinical symptoms indicating severe damage to the nervous system and itching were usually not observed (Verpoest et al., 2018).

Scientists who are studying Aujeszky's disease have described in detail the pathological and anatomical changes during the course of this disease in pigs (Caruso et al., 2018; Wu et al., 2018; Zhao et al., 1996), but there is little data on microscopic changes of internal organs of suckling piglets that died from Aujeszky's disease, therefore we studied microscopic changes in the lungs, 
stomach, intestines, liver, pancreas, kidneys, thymus, lymph nodes, spleen, brain and spinal cord of sucking piglets that died from Aujeszky's disease. The study of histological changes in the internal organs of suckling piglets, due to the significant variability of the etiological factor of this disease, namely Suid alphaherpesvirus 1 had the particular relevance (Minamiguchi et al., 2019; Moreno et al., 2015).

\section{Materials and Methods}

For histological studies, we sampled the specimens of suckling piglets internal organs: lungs, stomach, intestines, liver, pancreas, kidneys, thymus, lymph nodes, spleen, brain and spinal cord that died from Aujeszky's disease.

The campling was carried out no later than 1 hour after the piglets death. The selected material was fixed in a $10 \%$ solution of neutral formalin, followed by treatment in ethanol of increasing concentration and finally was embedded in paraffin through chloroform. Sections with a thickness of 7-9 $\mu \mathrm{m}$ were obtained on a sled microtome, and stained with Karatsu hematoxylin and eosin. The preparations were examined using a binocular light microscope (Micros 100 LED) with magnifications ranging from $80 x$ to 200x. Photographing of the histopreparation was performed with a Canon EOS 550 D digital camera.

To study of microscopic changes during the coarse of Aujeszky's disease, we conducted microscopic examinations of the internal organs of dead piglets. Organ tissue samples were taken from 5 suckling piglets ( 4 to 6 days old). In all piglets, the diagnosis was confirmed by polymerase chain reaction (PCR).

Detection of DNA fragmetns of the Aujeszky disease virus was performed by PCR using «TagMan» system reagents. For the detection of the virus, primers were designed to detect the glycoprotein gB. The primers were selected in accordance with the optimal size, content of G-C pairs, avoiding large areas consisting of a single base and motifs that are repeated. Two pairs of primers were used and two consecutive reactions were carried out, the second pair of primers amplifies the DNA region inside the product of the first reaction. The primers were tested with the Vector NTI Advance 11 software using Gene Bank data and validated against commercial standards by Veterinary Diagnostics Center LLC.

Isolation of viral DNA from pathological material samples was performed using the MagMAX ${ }^{\text {тм }}$ CORE Nucleic Acid Purification Kit, Thermo Fisher, Germany.

\section{Results and Discussion}

A postmortem examination of 5 suckling piglets aged 4 - 6 days that died from Aujeszky's disease was carried out. In all cases the diagnosis was confirmed by PCR.

During the postmortem examination it was found that all the lymph nodes were clearly enlarged, reddish-brown in color. The thymus is swollen, flaccid, pinkish. The lungs are unevenly colored, with patches of pink and red of different sizes. Interlobular connective tissue is unevenly edematous. The stomach was dilated. There were no macroscopic changes in it. Signs of serous catharral inflamation were recorded in the small intestine, cecum, and colon. The pancreas in all the piglets was edematous and had a pinkish color. There were no macroscopically significant changes in the spleen. The liver and kidneys from the surface had an uneven vascularization. In the brain and spinal cord, the blood vessels of the pia mater were dilated, overflowing with blood, hyperemia of the substance of the brain and spinal cord, and in the brain there were also swollen twists (Figure 1).

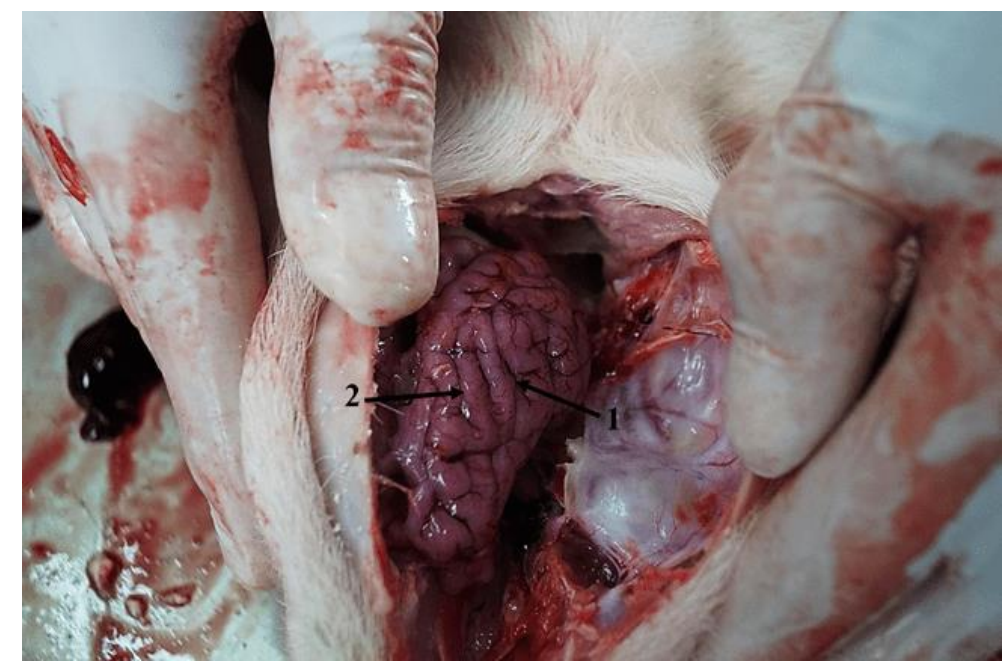

Figure 1. The brain of a 4-day-old piglet died from Aujeszky's disease: 1 - Dilated, blood vessels of the pia mater filled with blood; 2 - Edema of the convolutions of the brain and hyperemia of the brain substance.

During the histological studies of the lungs, we established that the interlobular fibrouse tissue was edematous. All blood vessels were dilated, replenished with blood cells. It was registered the destruction of endotelial cells and edema, granular distrophy and destruction of tunica media was recorded.

The walls of the alveoli were unevenly thickened due to the dilatation blood capillaries with overflow with blood and infiltration with blood cells - erythrocytes, lymphocytes and monocytes. Some of the erythrocytes were destroyed. The epithelium was absent in all alveoli. There were isolated partially destroyed type II pneumocytes with intranuclear eosinophilic inclusion bodies near the walls of the some alveoli. In the lung tissue it was detected eosinofilic corpuscular insertions in nuclei of lymphocytes, monocytes and macrophages (Figure 2). 


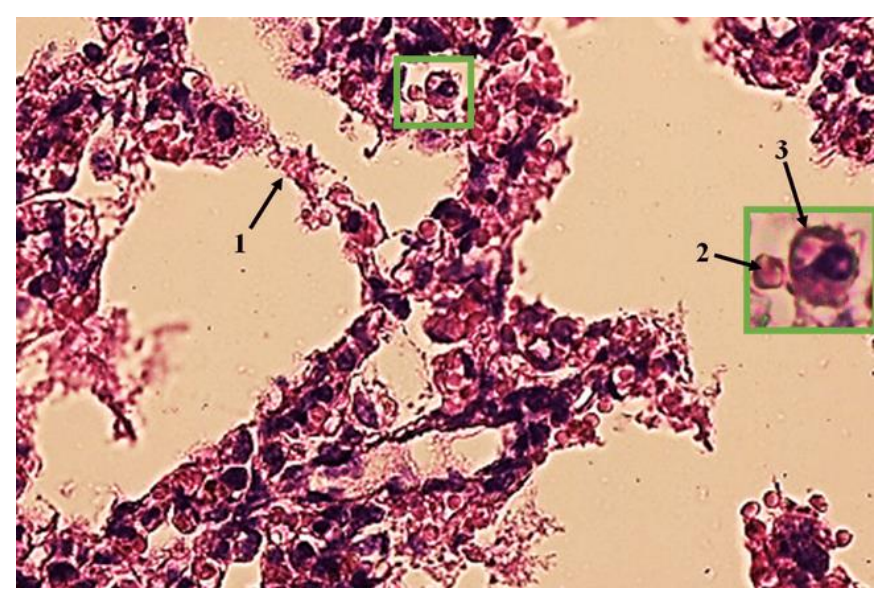

Figure 2. Lungs of a pig died from Aujeszky's disease: 1 - necrosis and destruction of the alveoli wall cells; 2 - erythrocyte with focal hypochromic content; 3 - macrophage with phagocytosed eosinophilic substance and intranuclear eosinophilic corpuscular insertions. Hematoxylin Carazzi and eosin, 100x.

Around the bronchioles and bronchi an uneven edema was detected. In the lumen of a part of the bronchi and bronchioles an erythrocytes and epithelial cells were found at different stages of destruction. Eosinophilic corpuscular insertions were found in the nuclei of some of the epithelial cells. The mucous membrane of the large bronchi was partially edematous and its blood vessels were dilated and filled with blood. The partially destructed epithelial cells were detected in all bronchi. Intranuclear eosinophilic corpuscular insertions were found in segregated bronchial epithelial cells of various size (Figure 3). Some of these epithelial cells were detached into the bronchial lumen. Smooth muscle cells of the muscle plate of the bronchial mucosa were in a state of granular degeneration. The submucosis base was distinctly edematousand the part of its cells were destroyed.

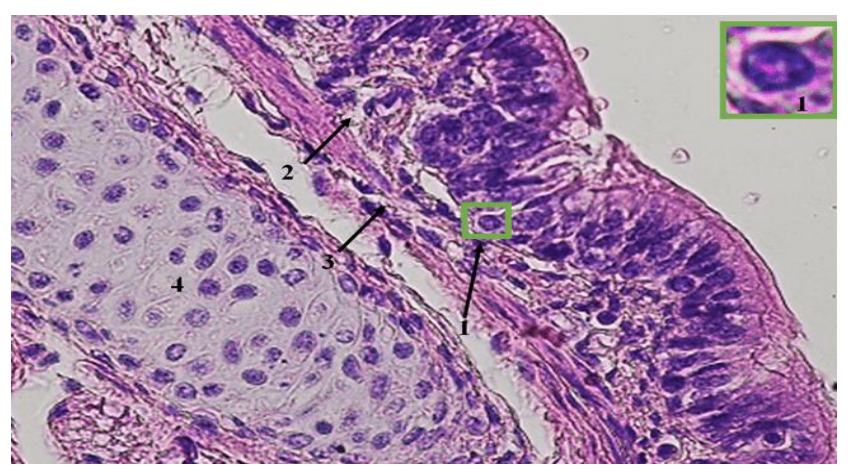

Figure 3. Lungs of a pig that died from Aujeszky's disease: 1 - epithelial cell of a large bronchus with an eosinophilic corpuscular insertions in the nucleus; 2 - edema of the mucous membrane, 3 - destruction of the cells of the muscle plate of the mucous membrane; 4 - the cartilage tissue is not changed. Hematoxylin Carazzi and eosin, $800 x$.

In the stomach, the upper part of the gastric dimples collapsed. At the same time, their middle and lower parts have got significant disorganization as a result of the destruction of the main and additional cells. Eosinophilic inclusion bodies were found in the nuclei of epithelial cells of all types (Figure 4). Locally in the gastric dimples, cellular syncytia appeared, which were one large, distinctly oxyphilic cytoplasm, in which 2 to 5 nuclei were expressed (Figure 5). Some of the nuclei of such cellular syncytia contained eosinophilic corpuscular insertions. The mucous membrane was infiltrated with single lymphocytes and monocytes. Some of these cells contained intranuclear eosinophilic inclusion bodies. The submucosa was distinctly edematous. in the muscular membrane of the stomach it was detected the oedema, granular degeneration of smooth muscle cells a. Some of the dystrophically altered cells were destroyed.

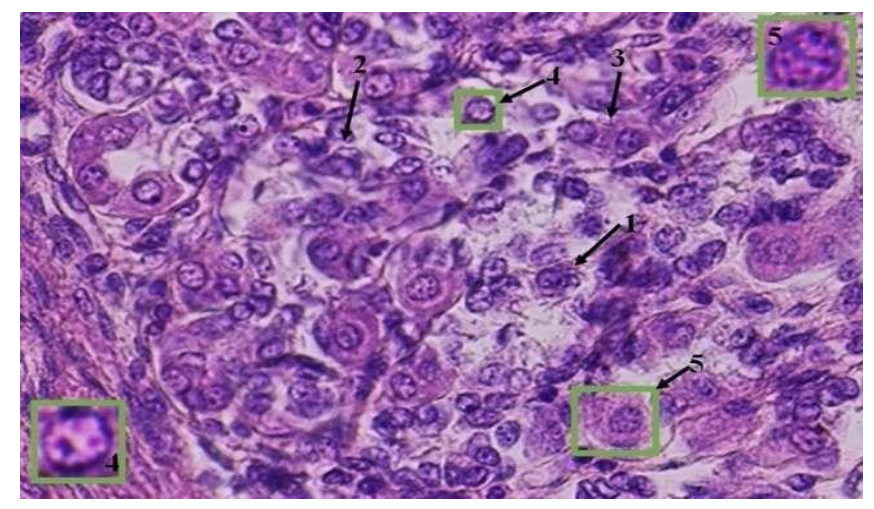

Figure 4. The stomach of a piglet at the age of 5 days, died from Aujeszky's disease: 1 - the disorganization of the gastric fossa; 2 - destruction of the main and additional cells; 3 - destruction of the parietal cell; 4 - eosinophilic corpuscular insertions in the nucleus of the main cell; 5 - predominantly eosinophilic nucleus of the parietal cell. Hematoxylin Carazzi and eosin, 400x. 


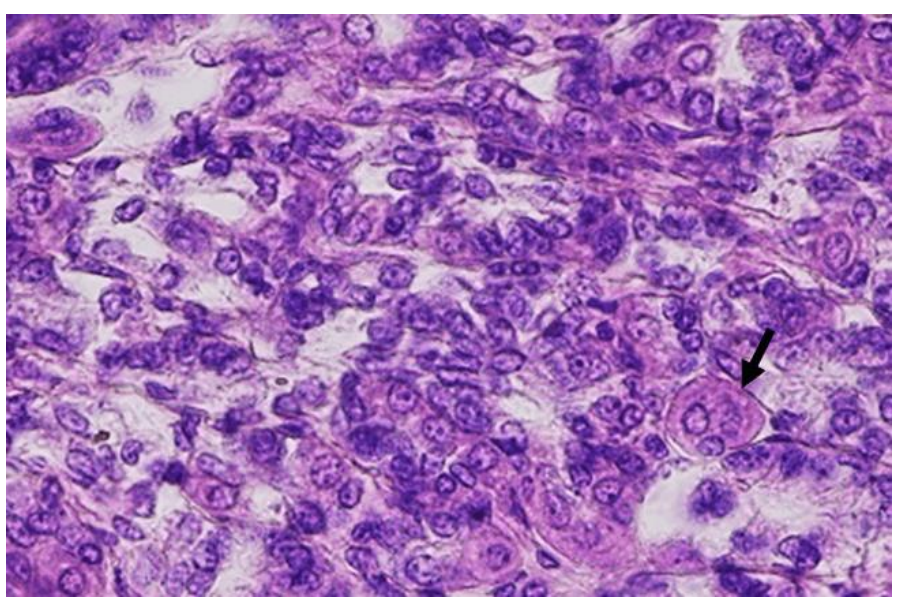

Figure 5. Stomach of a pig at the age of 6 days, died from Aujeszky's disease: syncytium of epithelial cells (shown by an arrow). Hematoxylin Carazzi and eosin, 400x.

Destruction of the villi was observed in all parts of the small intestine. The mucous membrane was edematous, infiltrated with lymphocytes, monocytes and a noticeable number of eosinophils. Some of the villus enterocytes, including those at different stages of destruction, contained intranuclear eosinophilic inclusion bodies (Figure 6). The submucosa was distinctly edematous. Smooth muscle cells of the muscular membrane were in a state of granular degeneration. Some of the dystrophically altered cells were destroyed. Selected areas of the small intestine showed expressive metaplasia of smooth muscle cells of the muscular membrane and mesotheliocytic cells of the serous membrane (Figure 7).

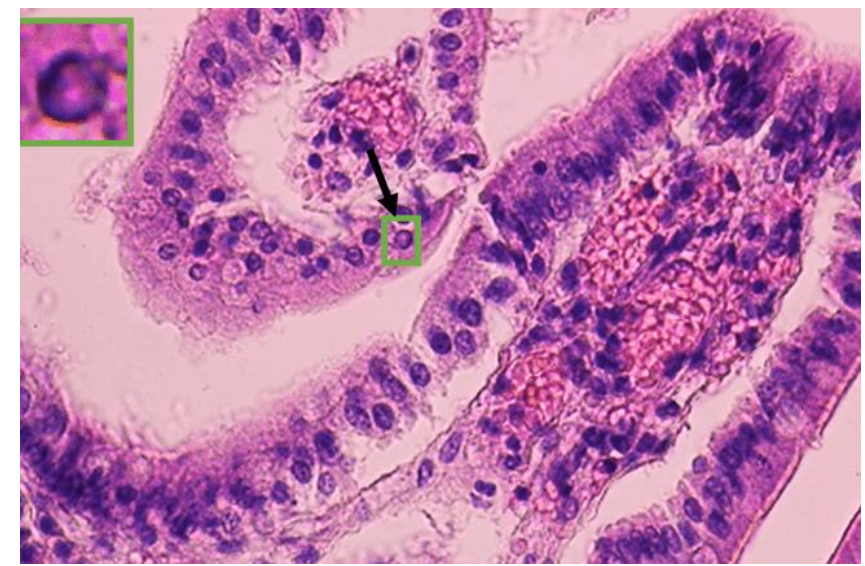

Figure 6. The jejunum of a 4-day-old pig that died from Aujeszky's disease: almost completely eosinophilic nucleus of the villus enterocyte (pointed). Hematoxylin Carazzi and eosin, 400x.

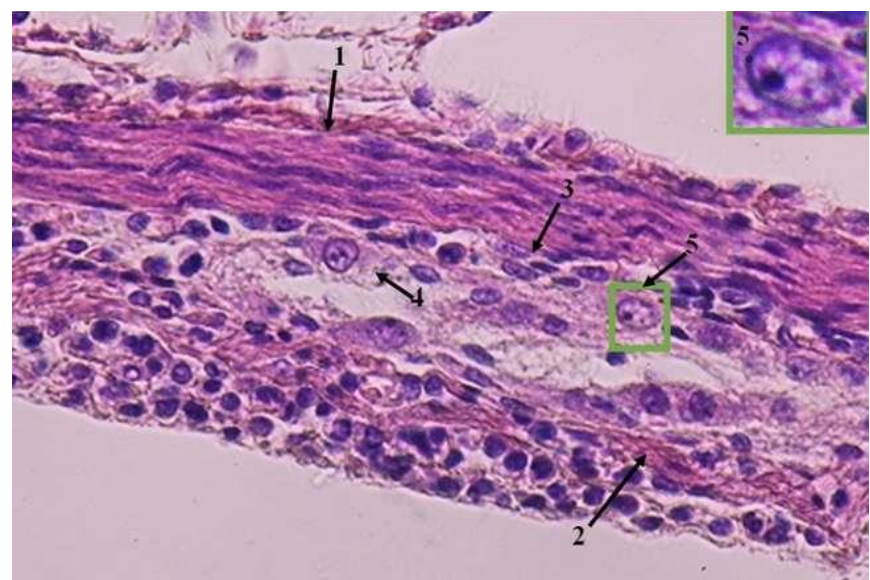

Figure 7. The ileum of a piglet at the age of 5 days, died from Aujeszky's disease: 1 - granular degeneration of smooth myocytes of the inner layer of the muscular membrane; 2 - granular dystrophy of smooth myocytes of the outer layer of the muscular membrane, 3 - the initial stages of metaplasia of smooth myocytes; 4 - the final stage of smooth muscle myocytes metaplasia; 5 intranuclear eosinophilic corpuscular insertions of a metaplastic cell; 6 - metaplasia of the mesotheliocyte. Hematoxylin Carazzi and eosin, 400x.

During the process of metaplasia of smooth myocytes at first stage the nucleus significantly increased getting an oval and then a round shape. With an increasing of nuclei size the character of staining had also changed. They became less basophilic clearly enlightened and eosinophilic corpuscular insertions were detected in cells (Figure 7). As well as nucleus the cytoplasm volume also increased in size becaming foamy and loosing its clear boundaries. The cytoplasm of the cells was unevenly stained by eosin. 
In most parts of the cecum and colon, destruction of the upper part of the crypts and disorganization of their middle and lower parts were recorded. In places the crypts were destroyed to their bottom. Eosinophilic corpuscular insertions were found in the nuclei of some epithelial cells (Figure 8). The mucous membrane was distinctly oedematous, unevenly infiltrated with lymphocytes and monocytes. In some places, expressive fibroblast hypertrophy was registered with forming of giant spindle-shaped cells with a big round or oval nucleus (Figure 8). The submucosa and muscular membranes of the cecum and colon were clearly swollen. Smooth myocytes of the muscular membrane were granular degenerated with partial destruction of cells.

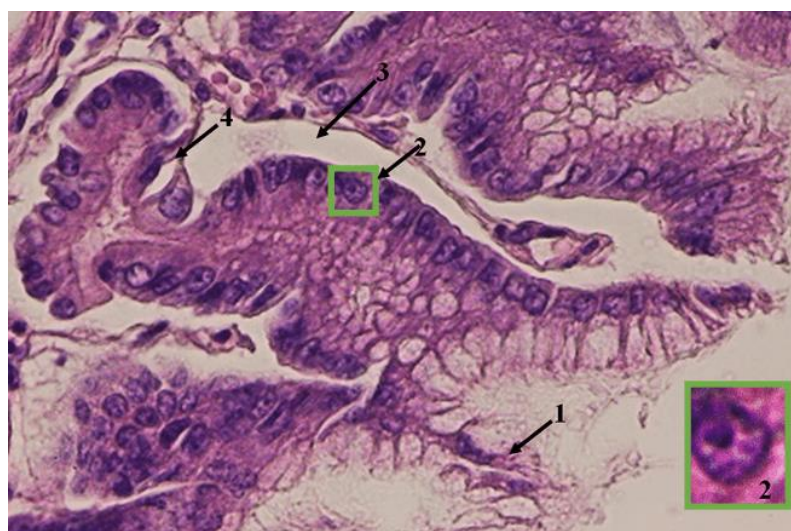

Figure 8. Crypts of the cecum of a 4-day-old piglet that died from Aujeszky's disease: 1 - destruction of the upper part of the crypt; 2 - intranuclear eosinophilic corpuscular insertions in the nucleus of the enetrocyte; 3 - subepithelial oedema; 4 - hypertrophy of fibroblast. Hematoxylin Carazzi and eosin, 400x.

A manifestating oedema in the thymus was registered. There was not detected clear border between cortex and medulla. A significant part of the lymphocytes number was in the state of necrotic decomposition. The thymic bodies were registered at different stages of decomposition. The cortex and medulla were unevenly swollen in all samples. Many lymphocytes in the cortex and medulla were necrotic and were at various stages of destruction. Significant deposits of hemosiderin appeared in the cortex. Eosinophilic corpuscular insertions were found in the nuclei of some lymphocytes (Figure 9).

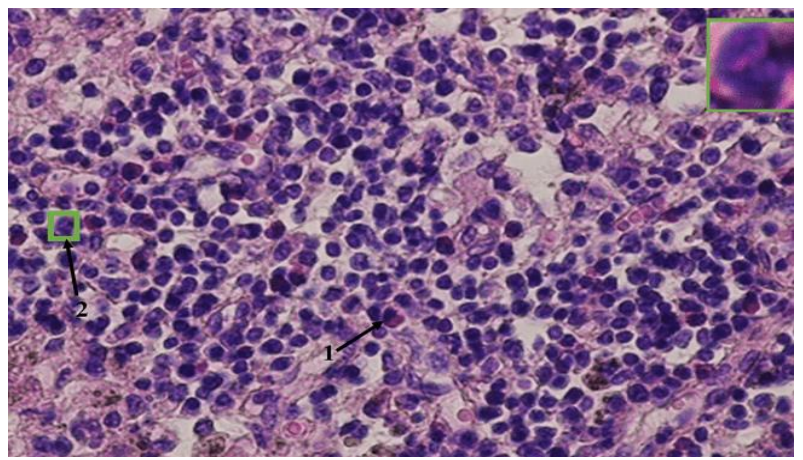

Figure 9. The medulla of the prescapular lymph node of a piglet died from Aujeszky's disease of 5 days after birth: 1 - eosinophils; 2 - eosinophilic corpuscular insertions in the nucleus of the lymphocyte. Hematoxylin Carazzi and eosin, 400x.

The red pulp of the spleen was unevenly infiltrated with lymphocytes and monocytes. Eosinophilic corpuscular insertions were detected in the nuclei of these cells. The size of the lymphoid nodules was greatly reduced. Microscopic changes in the brain and spinal cord were similar. The brains pia mater was edematous and its blood vessels were significantly dilated and filled with blood. There was also oedema between the pia mater and the brain matter. The blood vessels in the brain were clearly dilated and overflowing with blood. In some of the blood vessels stagnation of lymphocytes was recorded, and in some the release of lymphocytes outside the blood vessels. Glia was unevenly edematous and perivascular edema and edema around nerve cells and glial cells were also recorded. Some of the nervous cells were in a state of basophilia with some cells under necrotic decomposition or destruction (Figure 10). It was detected the neuronophagy and large glial nodules in some spots (Figure 11).

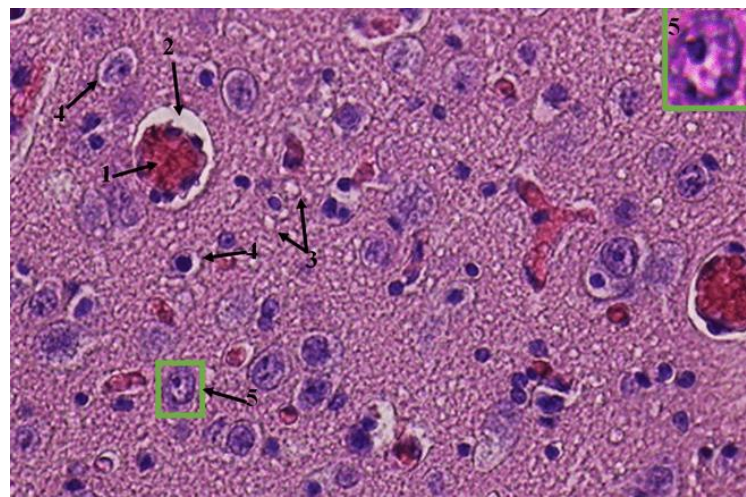

Figure 10. The brain of a 4-day-old piglet that died from Aujeszky's disease: 1 - an enlarged blood vessel filled with blood; 2 perivascular oedema; 3 - glial oedema; 4 - pericellular oedema; 5 - eosinophilic corpuscular insertions in the nucleus of the neurocytes. Hematoxylin Carazzi and eosin, 400x. 


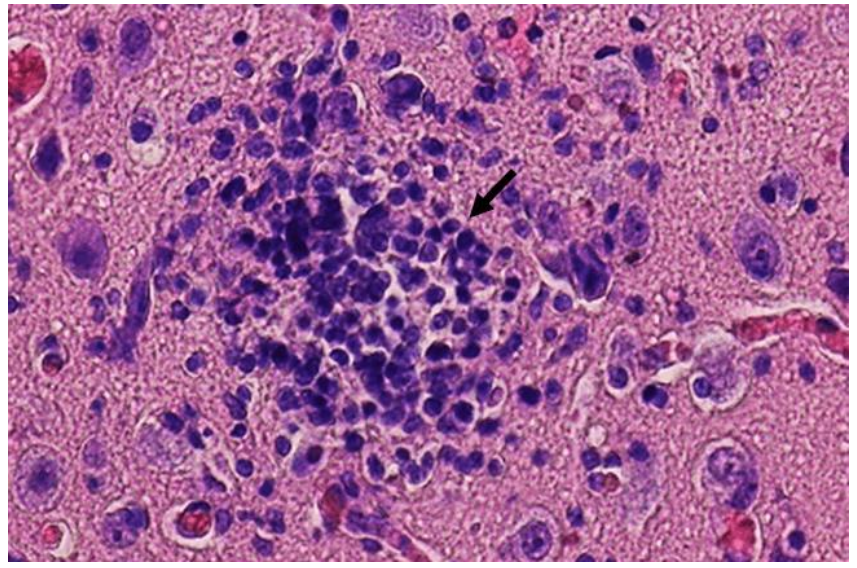

Figure 11. The brain of a 5-day-old piglet that died from Aujeszky's disease: 1 - granulomatosis (pointed with arrow). Hematoxylin Carazzi and eosin, 400x.

Eosinophilic inclusion bodies were found in the nuclei of some nervous cells (Figure 10). The nuclei of individual nervous cells became almost completely eosinophilic. The boundary between the cytoplasm and the surrounding glia of the cells was indistinct or non- manifestated. In the liver, the central veins of all hepatic lobules were enlarged and filled with blood cells. There was a slight swelling around these veins, and some of them had small granulomas, consisting of lymphocytes and single monocytes. Some granulomas also appeared to be distant from the central veins, but their amount was insignificant. Most of the hepatocytes of the hepatic lobules were in a state of granular degeneration (Figure 12).

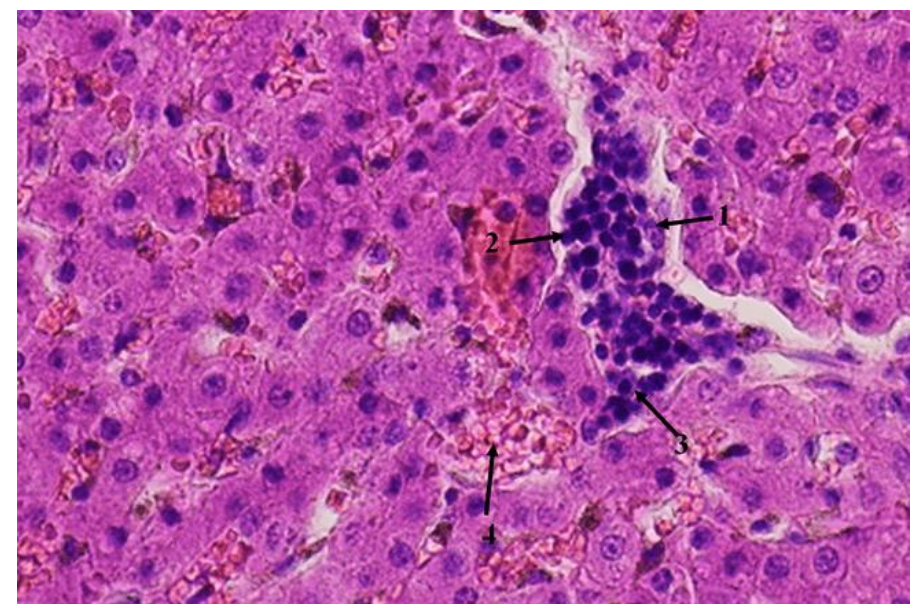

Figure 12. The Liver of a piglet at the age of 6 days, died from Aujeszky's disease: 1 - granuloma; 2 - lymphocyte; 3 - monocyte; 4 - destruction of erythrocytes. Hematoxylin Carazzi and eosin, 400x.

There was detected a large number of erythrocytes in the sinusoidal capillaries and most of them were at various stages of destruction. As a result of this destruction, hemosiderin sediments were detected in different spots. Part of this hemosiderin was phagocytosed by Kupffer's cells transformed into ordinary siderophages. In the hepatic triads it was detected dilated blood-filled veins and unevenly narrowed arteries and bile ducts. The stroma of the pancreas was clearly edematous, some of the particles were completely or partially necrotically decomposed. The vast majority of acini were disorganized, the homogeneous pole in all pancreatocytes was not differentiated. Most cells of all types in all pancreatic islets were in a state of granular degeneration. Some of the degenerated cells were destroyed (Figure 13).

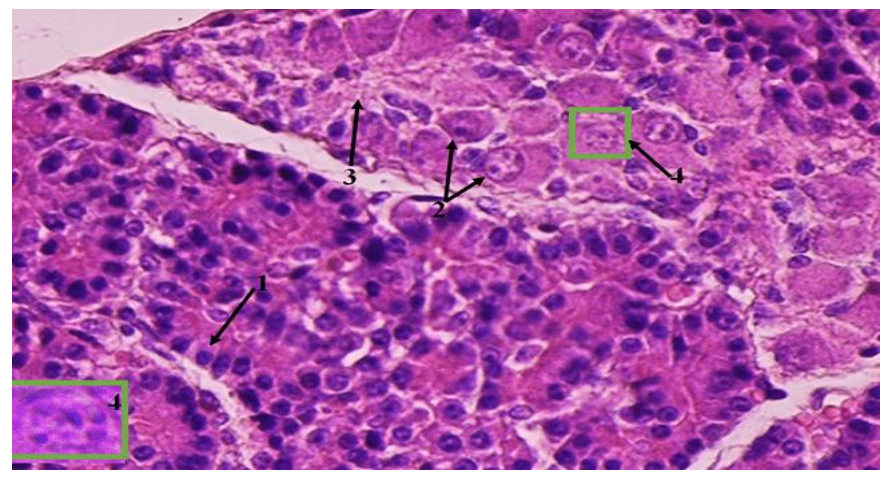

Figure 13. The pancreas of a piglet died from Aujeszky's disease at the age of 6 days: 1 - the absence of a homogeneous pole of the pancreatocyte; 2 - pancreatocyte metaplasia; 3 - destruction of metaplastic pancreatocyte; 4 - the nucleus of a metaplastic pancreatocyte with eosinophilic corpuscular insertions. Hematoxylin Carazzi and eosin, 400x. 
The pancreatocytic metaplasia of the part of the pancreatic islets was revealed. These structures were consisted of large transformed cells of various shapes with a large, light round nucleus located at one of the poles of the cell. The entire cytoplasm of these cells was filled with eosin with the same intensity with some stain differentiation at homogeneous poles. Some of the metaplastic cells were destroyed. In the nuclei of a part of the metaplastic cells, eosinophilic corpuscular insertions were detected. At the same time, the nuclear membrane was poorly differentiated and in some areas it did not appear at all (Figure 13).

In the course of histological studies of all renal corpuscles, we found granular degeneration of glomerular mesangium kidney cells of Aujeszky's disease pig outbracks. In the convoluted tubules, granular degeneration of epithelial cells was recorded. A significant part of the dystrophic cells were destroyed. There was subepithelial edema of varying intensity in different investigated kidney areas. In the area of the rectal tubules, the stroma of the kidney was pronounced edematous and significant subepithelial edema was recorded. Some amount of the tubules showed granular degeneration of the epithelial cells. In major part of the tubules its epithelium layer was completely or partially destroyed.

\section{Conclusion}

As the result of studies it was revealed significant pathomorphological changes in most internal organs as a result of alterning impact of the Aujeszky's disease virus onto pig organism. As a result of histological studies of internal organs (lungs, stomach, intestines, liver, pancreas, kidneys, thymus, lymph nodes, spleen, brain and spinal cord) of piglets that died due to Aujeszky's disease we found significant microscopic changes in all morphological formations of organism.

A significant negative effect of the virus onto body ecology integrity was revealed.

We have described a large number of micromorphological signs of pathological changes in piglets' Aujeszky's disease and identified a set of criteria that can be used to substantiate the postmortem diagnosis of this disease.

\section{References}

Cano-Terriza, D., Martínez, R., Moreno, A., Pérez-Marín, J. E., Jiménez-Ruiz, S., Paniagua, J., Borge, C., García-Bocanegra, I. (2019). Survey of Aujeszky's Disease Virus in Hunting Dogs from Spain. EcoHealth, 16(2), 351-355.

Caruso, C., Vitale, N., Prato, R., Radaelli, M. C., Zoppi, S., Possidente, R., Dondo, A., Chiavacci, L., Moreno Martin, A. M., Masoero, L. (2018). Pseudorabies virus in North-West Italian wild boar (Sus scrofa) populations: prevalence and risk factors to support a territorial risk-based surveillance. Veterinaria italiana, 54(4), 337-341.

Deblanc, C., Oger, A., Simon, G., Le Potier, M. F. (2019). Genetic Diversity among Pseudorabies Viruses Isolated from Dogs in France from 2006 to 2018. Pathogens (Basel, Switzerland), 8(4), 266.

Dong, J., Bai, J., Sun, T., Gu, Z., Wang, J., Sun, H., Jiang, P. (2017). Comparative pathogenicity and immunogenicity of triple and double gene-deletion pseudorabies virus vaccine candidates. Research in veterinary science, 115, 17-23.

Gu, J., Hu, D., Peng, T., Wang, Y., Ma, Z., Liu, Z., Meng, F., Shang, Y., Liu, S., Xiao, Y. (2018). Epidemiological investigation of pseudorabies in Shandong Province from 2013 to 2016. Transboundary and emerging diseases, 65(3), 890-898.

Guan, X., Liu, J., Jiang, H., Wu, C. X., Chen, H. C., Liu, Z. F. (2018). Expression of pseudorabies virus-encoded long noncoding RNAs in epithelial cells and neurons. Journal of neurovirology, 24(5), 597-605.

Lai, I. H., Chang, C. D., Shih, W. L. (2019). Apoptosis Induction by Pseudorabies Virus via Oxidative Stress and Subsequent DNA Damage Signaling. Intervirology, 62(3-4), 116-123.

Lane, M. A., Lee, K. Z., Salazar, K., O'Steen, B. E., Bloom, D. C., Fuller, D. D., Reier, P. J. (2012). Respiratory function following bilateral mid-cervical contusion injury in the adult rat. Experimental neurology, 235(1), 197-210.

Laval, K., Vernejoul, J. B., Van Cleemput, J., Koyuncu, O. O., Enquist, L. W. (2018). Virulent Pseudorabies Virus Infection Induces a Specific and Lethal Systemic Inflammatory Response in Mice. Journal of virology, 92(24), 1614-1618.

Liu, Y., Chen, Q., Rao, X., Diao, X., Yang, L., Fang, X., Hogeveen, H. (2019). An economic assessment of pseudorabies (Aujeszky' disease) elimination on hog farms in China. Preventive veterinary medicine, 163, 24-30.

Miller, J. L., Weed, D. J., Lee, B. H., Pritchard, S. M., Nicola, A. V. (2019). Low-pH Endocytic Entry of the Porcine Alphaherpesvirus Pseudorabies Virus. Journal of virology, 93(2).

Minamiguchi, K., Kojima, S., Sakumoto, K., Kirisawa, R. (2019). Isolation and molecular characterization of a variant of Chinese gCgenotype II pseudorabies virus from a hunting dog infected by biting a wild boar in Japan and its pathogenicity in a mouse model. Virus genes, 55(3), 322-331.

Moreno, A., Sozzi, E., Grilli, G., Gibelli, L. R., Gelmetti, D., Lelli, D., Chiari, M., Prati, P., Alborali, G. L., Boniotti, M. B., Lavazza, A., Cordioli, P. (2015). Detection and molecular analysis of Pseudorabies virus strains isolated from dogs and a wild boar in Italy. Veterinary Microbiology, 177(3-4), 359-365.

Sorooshyari, S. K., Taylor, M. P., Poor, H. V. (2018). Probabilistic Modeling of Pseudorabies Virus Infection in a Neural Circuit. Journal of computational biology, 25(11), 1231-1246.

Tong, C., Chen, Z., Liu, F., Qiao, Y., Chen, T., Wang, X. (2020). Antiviral activities of Radix isatidis polysaccharide against pseudorabies virus in swine testicle cells. BMC complementary medicine and therapies, 20(1), 48.

Tong, W., Liu, F., Zheng, H., Liang, C., Zhou, Y. J., Jiang, Y. F., Shan, T. L., Gao, F., Li, G. X., Tong, G. Z. (2015). Emergence of a Pseudorabies virus variant with increased virulence to piglets. Veterinary Microbiology, 181(3-4), 236-240.

Ukhovskyi, V. V., Drozhzhe, Z. M., Bezymennyi, M. V., Mezhenska, N. A., Romanov, O. M., Karpulenko, M. S., Zhovnir, O. M., Mezhensky, A. O. (2020). Ecological and geographical characteristics of Aujeszky's disease among the wild pigs in Ukraine. Ukrainian Journal of Ecology, 10 (2), 45-49.

Verpoest, S., Cay, A. B., Van Campe, W., Mostin, L., Welby, S., Favoree,I H., De Regge, N. (2016). Age- and strain-dependent differences in the outcome of experimental infections of domestic pigs with wild boar pseudorabies virus isolates. Journal of General Virology, 97(2), 487-495.

Verpoest, S., Redant, V., Cay, A. B., Favoreel, H., De Regge, N. (2018). Reduced virulence of a pseudorabies virus isolate from wild boar origin in domestic pigs correlates with hampered visceral spread and age-dependent reduced neuroinvasive capacity. Virulence, 9(1), 149-162.

Wong, G., Lu, J., Zhang, W., Gao, G. F. (2019). Pseudorabies virus: a neglected zoonotic pathogen in humans? Emerging microbes \& infections, 8(1), 150-154.

Wu, Q., Zhang, H., Dong, H., Mehmood, K., Chang, Z., Li, K., Liu, S., Rehman, M. U., Nabi, F., Javed, M. T., Zhu, H., Li, J. (2018). Seroprevalence and risk factors associated with Pseudorabies virus infection in Tibetan pigs in Tibet. BMC veterinary research, $14(1), 25$. 
Xia, L., Sun, Q., Wang, J., Chen, Q., Liu, P., Shen, C., Sun, J., Tu, Y., Shen, S., Zhu, J., Zhao, H., Wang, Q., Li, B., Tao, J., Soares Magalhaes, R. J., Yan, Y., Cai, C. (2018). Epidemiology of pseudorabies in intensive pig farms in Shanghai, China: Herd-level prevalence and risk factors. Preventive veterinary medicine, 159, 51-56.

Zhao, H., Wang, S., Liu, C., Han, J., Tang, J., Zhou, L., Ge, X., Guo, X., Yang, H. (2018). The pUL56 of pseudorabies virus variant induces downregulation of swine leukocyte antigen class I molecules through the lysosome pathway. Virus research, 251, 56-67. Zhao, X., Tong, W., Song, X., Jia, R., Li, L., Zou, Y., He, C., Liang, X., Lu, C., Jing, B., Lin, J., Yin, L., Ye, G., Yue, G., Wang, Y., Yin, Z. (2018). Antiviral Effect of Resveratrol in Piglets Infected with Virulent Pseudorabies Virus. Viruses, $10(9), 457$.

Zhao, Y., Narita, M., Kawashima, K. (1996). Pathologic changes in closed porcine intestinal loops inoculated with Aujeszky's disease virus, 58(8), 809-810.

\section{Citation:}

Ukhovskyi, V.V., Romanov, O.M., Borisevich, B.V., Tarasov, O.A., Ukhovska, T.M., Pyskun, A.V., Solomon, V.V., Ayshpur, O.Y. (2020). Pathologic changes in pig organs, infected with the Aujeszky's disease. Ukrainian Journal of Ecology, 10(5), 8-15. 